Ansicht, setzt das Zurückbehaltungsrecht nach $₫ 273$ Abs. 1 BGB keine unmittelbare Gefahr für Leben oder Gesundheit voraus. Gleichwohl soll bei geringfügigen oder kurzfristigen Verstößen des Arbeitgebers gegen Arbeitsschutzpflichten, die keinen nachhaltigen Schaden beim Mitarbeiter bewirken können, kein Zurückbehaltungsrecht ausgeübt werden können ${ }^{72}$. Das Risiko sich mit dem Corona-Virus SARS-CoV-2 zu infizieren ist ein unmittelbares Lebens- bzw. Gesundheitsrisiko, sodass ein unzureichender Schutz hiergegen das Krankenhauspersonal zu einem Zurückbehaltungsrecht nach $\$ 273$ Abs. 1 BGB berechtigt ${ }^{73}$. Falls der Arbeitnehmer tatsächlich von seinem Zurückbehaltungsrecht Gebrauch macht - und zur Ausübung dieses Rechts berechtigt ist -, gerät der Arbeitgeber in Annahmeverzug, mit der Folge, dass der Arbeitnehmer seines Vergütungsanspruchs nicht verlustig wird ${ }^{74}$.

\section{Fazit}

Die relativ plötzlich auftretende COVID-19-Pandemie stellt die Akteure im deutschen Gesundheitswesen vor große Herausforderungen. Insbesondere Krankenhäuser waren zu Beginn der Pandemie gezwungen - zunächst vornehmlich aufgrund der behördlichen Vorgabe elektive Eingriffe abzusagen -, ihre Personaleinsatzplanung von heute auf morgen zu ändern. Nahezu jedes Krankenhaus hat sein Schichtsystem auf ein 12-Stunden-Schichtsystem umgestellt und eine Kohortierung durchgeführt, um bei der Versorgung von SARS-CoV-2-infizierten Patienten eine organisatorische und räumliche Trennung des zugewiesenen Personals zu erreichen. Derartige Maßnahmen sind nach den Ausnahmeregelungen der \$\$14, 15 ArbZG zulässig. Sie unterliegen zudem der Mitbestimmung des Betriebsrates nach $₫ 87$ Abs. 1 Nr. 2 BetrVG. Am Corona-
Virus SARS-CoV-2 erkrankte Mitarbeiter medizinischer Einrichtungen haben Anspruch auf Entgeltfortzahlung nach $\$ 3$ Abs. 3 EFZG. Krankenhauspersonal, welches unter amtlich angeordneter Quarantäne steht oder dem sogenannten beruflichen Beschäftigungsverbot nach dem Infektionsschutzgesetz unterliegt, ist von seiner Arbeitsverpflichtung befreit. Hier greift der Entschädigungsanspruch gegenüber dem Staat nach $\$ 56$ Abs. 1 IfSG. Um jedoch den effektiven Einsatz medizinischen Personals - verständlicherweise auch nach dem Kontakt mit einem SARS-CoV-2-infizierten Patienten - zu ermöglichen, hat das Robert Koch-Institut Empfehlungen zum Management von Kontaktpersonen veröffentlicht. Diese Empfehlungen verdeutlichen, wie wichtig die Einhaltung der Arbeitsschutzmaßnahmen im Zuge der COVID-19-Pandemie ist. Kommt der Krankenhausträger diesen Maßnahmen nicht oder nicht ausreichend nach, steht dem Mitarbeiter ein Zurückbehaltungsrecht an seiner Arbeitsleistung nach $\$ 273$ Abs. 1 BGB zu. Elementare Voraussetzung, damit ein Krankenhaus seine Arbeitsschutzmaßnahmen erfüllen kann, ist jedoch die ausreichende Ausstattung mit Schutzausrüstung wie Atemschutzmasken, Desinfektionsmitteln, Einweghandschuhen sowie Schutzkitteln. Bedauerlicherweise ist die Versorgung mit diesen Materialien - entgegen anderslautender politischer Verlautbarungen - alles andere als gewährleistet.

72) Blomeyer, in: MHdB ArbR, 4. Aufl. 2018, \$41, Rdnrn. 15, 16; Reinfeld, in: MAH ArbR, 4. Aufl. 2017, \$34, Rdnr. 13.

73) Allerdings ist wichtig zu betonen, dass auch im Falle einer drohenden Infizierungsgefahr die Arbeitspflicht nach wie vor fortbesteht. Das bestehende Risiko einer Infizierung rechtfertigt es nicht, die Arbeitsleistung zurückzuhalten - nur die unzureichenden Schutzmaßnahmen.

74) Reinfeld, in: MAH ArbR, 4. Aufl. 2017, §34, Rdnr. 13.

\title{
Rechtliche Betreuung in Zeiten der COVID-19 Pandemie
}

\section{Anna Schwedler und Sarah Glaab}

\begin{abstract}
Ältere Menschen und Menschen mit Vorerkrankungen bedürfen eines besonderen Schutzes vor einer Infektion mit COVID-19. Dieser Schutz gestaltet sich letztlich so, dass sich die Menschen in das Private zurückziehen und den Kontakt $\mathrm{zu}$ Anderen weitgehend meiden sollen. Allerdings können vor allem ältere Menschen eine besondere Unterstïtzung bei Erledigung ihrer Angelegenheiten benötigen, die im Hinblick auf die Teilhabe im Rechtsverkehr häufig durch eine rechtliche Betreuung sichergestellt wird. Dafür ist grundsätzlich der persönliche Kontakt zwischen den Betroffenen, den Betreuern, den Betreuungsgerichten, den Sachverständigen und den Verfahrenspflegern erforderlich. Es fragt sich daher, welche Auswirkungen die Maßnahmen zur Eindämmung des Coronavirus auf das betreuungsrechtliche Verfahren und das bestehende Betreuungsverhältnis haben.
\end{abstract}

Dr. iur. Anna Schwedler und Ass. iur. Sarah Glaab,

Institut für Zivilrecht und Wirtschaftsrecht,

Fachbereich Rechtswissenschaft,

Goethe-Universität Frankfurt,

Theodor-W.-Adorno-Platz 4,

60629 Frankfurt (Postfach 29), Deutschland

\section{Das Verfahren der Betreuerbestellung}

Die landesrechtlichen Maßnahmen zur Eindämmung der COVID-19 Pandemie scheinen insbesondere die Betreuungsgerichte vor neue Herausforderungen zu stellen, da die verfahrensrechtlichen Regelungen vor allem den persönlichen Kontakt zwischen den Beteiligten voraussetzen. Aufgrund der Auswirkungen auf das Selbstbestimmungsrecht des Betroffenen sind spezielle prozessuale Sicherungen vorgesehen, wie Beteiligungsbefugnisse, Anhörungsrechte und eine Pflicht zur sachverständigen Begutachtung, die allesamt mit persönlichen Interaktionen verbunden sind.

Im Vordergrund des Betreuungsverfahrens steht die Prüfung ob bzw. in welchem Umfang der Betroffene seine Angelegenheiten tatsächlich nicht mehr selbst oder durch Bevollmächtigte besorgen kann. Eine Betreuung darf schließlich nur dann eingerichtet werden, wenn und soweit sie erforderlich ist, vgl. \1896 BGB. Anhand dieses Erforderlichkeitskriteriums lässt sich auch die Ausgestaltung des Betreuungsverfahrens erklären, das eine Reihe von Verfahrensvorschriften vorsieht, um die Rechte des Betroffenen zu wahren. 


\section{Anhörung des Betroffenen}

Vor der Bestellung des Betreuers sowie vor der Anordnung eines Einwilligungsvorbehalts hat das Gericht den Betroffenen persönlich anzuhören, $\$ 278$ Abs. 1 FamFG. Die Anhörung dient der Gewährung des rechtlichen Gehörs (Art. 103 Abs. 1 GG). Dabei differenziert das Verfahrensrecht zwischen einer Anhörung und dem Verschaffen des persönlichen Eindrucks, $\$ 278$ Abs. 1 S. 1, 2 FamFG. Normalerweise soll sich das Gericht den persönlichen Eindruck in der üblichen Umgebung (Wohnung, Pflegeheim) verschaffen, wenn es der Betroffene verlangt oder wenn es der Sachaufklärung dient und der Betroffene nicht widerspricht, \$278 Abs. 1 S. 3 FamFG. Im Rahmen der Anhörung ist mit dem Betroffenen dann zu erörtern, für welche Aufgabenkreise eine Betreuung in Betracht kommt und welche Person das Amt des Betreuers wahrnehmen bzw. nicht wahrnehmen soll, $₫ 278$ Abs. 2 S. 3 FamFG. In Bezug auf den Ort der Anhörung verbleibt dem Gericht ein Ermessensspielraum ${ }^{1}$. Der Betroffene darf gegen seinen Willen in seiner Wohnung weder angehört noch begutachtet werden ${ }^{2}$.

\section{a) Ausnahmen von der Anhörungspflicht}

Das Gesetz sieht vor, dass die Anhörung nach $\$ \$ 278$ Abs. 4, 34 Abs. 2 FamFG ausnahmsweise unterbleiben kann, wenn aufgrund der Anhörung zu befürchten ist, dass erhebliche Nachteile für die Gesundheit des Betroffenen eintreten können, oder der Betroffene offensichtlich nicht in der Lage ist, seinen Willen kundzutun. Diese Entscheidung darf indes nur auf Grundlage eines ärztlichen Gutachtens ergehen.

\section{b) Anhörung in Zeiten der COVID-19 Pandemie}

Inwiefern das Betreuungsgericht in Zeiten der COVID-19 Pandemie von einer Anhörung absehen kann, wird nicht einheitlich beurteilt. Vertreten wird zurzeit, dass von der Anhörung insgesamt abzusehen ist, um so den Schutz der Betroffenen und des erkennenden Gerichts vor einer Infektion zu vermeiden ${ }^{3}$. Die Anhörung müsse auch nicht nachgeholt werden ${ }^{4}$. Aufgrund der zentralen Bedeutung der Gewährung des rechtlichen Gehörs wird dieser pauschale Verzicht auf die Anhörung indes zu recht kritisch beleuchtet ${ }^{5}$. Die Anhörung dient nicht nur der Gewährung des Rechts auf rechtliches Gehör, sondern auch der Sachverhaltsermittlung. Die Ergebnisse dieser Ermittlungen sind wiederum für die Frage, ob und für welche Aufgabenbereiche eine Betreuung zu bestellen ist, zentral. Ebenso ist zu berücksichtigen, dass die Anhörung nicht zu einem längeren oder engen Kontakt zwischen den Betroffenen führt und hygienische Schutzmaßnahmen ergriffen werden können. Daher muss gelten, dass auf die Anhörung nicht pauschal verzichtet werden kann. Stattdessen muss es immer auf den Einzelfall ankommen. Ein Absehen von der Anhörung kann beispielsweise dann angebracht sein, wenn der Betroffene nachweislich mit dem Coronavirus infiziert ist und ein ausreichender Schutz für das erkennende Gericht nicht möglich ist. $\mathrm{Zu}$ überlegen ist dabei, inwiefern eine Anhörung mittels Videokonferenz entsprechend $\$ 128$ a ZPO im Rahmen der persönlichen Anhörung eingesetzt werden könnte. Dies setzt voraus, dass sowohl die Betreuungsgerichte als auch die Betroffenen mit dieser Technik ausgestattet sind und diese auch bedienen können. Darüber hinaus hat das Betreuungsgericht zu prüfen, ob eine Videokonferenz für den Fall der persönlichen Anhörung tatsächlich geeignet ist, vgl. $\$ 32$ Abs. 3 FamFG. Dies ist zum Beispiel zu verneinen, wenn der Betroffene stark schwerhörig ist oder wenn es für die weitere Sachverhaltsaufklärung erforderlich ist, die übliche Umgebung des Betroffenen genauer kennenzulernen.

\section{Bestellung eines Sachverständigengutachtens}

Grundsätzlich ist vor der Bestellung eines Betreuers oder der Anordnung eines Einwilligungsvorbehalts eine förmliche Beweisaufnahme durch Einholung eines Gutachtens über die Notwendigkeit der Maßnahme erforderlich. Eine Begutachtung nach Aktenlage ist grundsätzlich unzulässig ${ }^{6}$.

\section{a) Absehen von einer Begutachtung}

Unter welchen Voraussetzungen auf ein gerichtliches Sachverständigengutachten verzichtet werden kann, regeln die \\$2 $280 \mathrm{ff}$. FamFG. So kann ein ärztliches Zeugnis ausreichen, wenn der Betroffene selbst den Antrag auf Bestellung eines Betreuers gestellt und auf die Begutachtung durch einen Sachverständigen verzichtet hat und die Begutachtung im Hinblick auf die Aufgabenkreise unverhältnismäBig erscheint, $\int 281$ Abs. 1 Nr. 1 FamFG. Des Weiteren kann das Gericht im Verfahren zur Betreuerbestellung von der Einholung eines Gutachtens nach $\$ 280$ Abs. 1 FamFG absehen, soweit durch die Verwendung eines bestehenden ärztlichen Gutachtens des MDK nach $\$ 18$ SGB XI festgestellt werden kann, inwieweit bei dem Betroffenen infolge einer psychischen Krankheit oder einer geistigen oder seelischen Behinderung die Voraussetzungen für die Bestellung eines Betreuers vorliegen, $\$ 282$ Abs. 1 FamFG. Diese Regelung wird als bedenklich eingestuft ${ }^{7}$, da das Pflegegutachten in der Regel nur die bestehende Erkrankung bzw. Behinderung beschreibt und sich nicht mit der Frage der Notwendigkeit einer Betreuung befasst. Dennoch kann das Gericht von einer ergänzenden Begutachtung absehen, wenn die Informationen im Gutachten ausreichend sind, $\$ 282$ Abs. 4 FamFG. In diesem Fall ist die Einwilligung des Betroffenen oder seines Pflegers für das Verfahren einzuholen, $\$ 282$ Abs. 3 S. 1 FamFG.

b) Begutachtung in Zeiten von COVID-19

Es zeigt sich, dass die Hürden, die an den Verzicht gestellt werden, recht hoch sind. Allein das Vorhandensein einer möglichen Infektion mit dem Coronavirus sollte pauschal nicht dazu veranlassen, auf ein Sachverständigengutachten zu verzichten. Dies sollte je nach Einzelfall sorgfältig geprüft werden. Zu bedenken ist ja, dass die Sachverständigen selbstverständlich hygienische Schutzmaßnahmen ergreifen können und aufgrund ihres fachlichen Hintergrundes diese sicherlich auch gut beherrschen. Natürlich muss bei der Auswahl des Sachverständigen darauf geachtet werden, dass der Sachverständige nicht selbst infiziert ist und ggf. nicht selbst zur Risikogruppe zählt.

\section{Bestellung eines Verfahrenspflegers}

Das Gericht hat dem Betroffenen einen Verfahrenspfleger zu bestellen, wenn dies zur Wahrnehmung der Interessen des Betroffenen erforderlich erscheint, \276 Abs. 1 S. 1 FamFG. Dies ist in der Regel zu bejahen, wenn von der persönlichen Anhörung des Betroffenen nach $\$ \int 278$ Abs. 4, 34 Abs. 2 FamFG abgesehen werden soll, sei es weil von der Anhörung Gesundheitsgefahren für den Betroffenen ausgehen oder weil er gar nicht mehr in der Lage ist sich zu äußern ${ }^{8}$. Zum anderen ist die Erforderlichkeit eines Verfahrenspflegers regelmäßig dann zu bejahen, wenn ein

1) Budde, in: Keidel, 20. Aufl. 2020, $\$ 278$ FamFG, Rdnr. 7.

2) BGH, FamRZ 2013, 31

3) Grotkopp, FamRZ Beitrag 2020, 2; AG Brandenburg v. 6. 4.2020 -85 XVII 69/20, juris.

4) Grotkopp, FamRZ Beitrag 2020, 2.

5) Beckmann, FamRZ Beitrag 2020, $1 \mathrm{ff}$

6) BGH, FamRZ 2015, 486; BGH, NJW-RR 2018, 449

7) Schneider, in: MüKo/BGB, 8. Aufl. 2020, $\$ 1896$, Rdnr. 201

8) Schneider, in: MüKo/BGB, 8. Aufl. 2020, \$1896, Rdnr. 162. 
Betreuer zur Besorgung aller (bzw. fast aller ${ }^{9}$ ) Angelegenheiten des Betroffenen bestellt werden soll bzw. dies möglich erscheint ${ }^{10}$ oder dessen Aufgabenkreis entsprechend erweitert werden soll.

\section{a) Absehen von der Verfahrenspflegerbestellung}

Nach $\$ 276$ Abs. 2 FamFG kann von der Bestellung in diesen Fällen abgesehen werden, wenn ein Interesse des Betroffenen an der Bestellung des Verfahrenspflegers offensichtlich nicht besteht. Die Nichtbestellung ist zu begründen und sollte restriktiv angewendet werden ${ }^{11}$. In den sonstigen Fällen kommt es auf den Einzelfall an, insbesondere auf die Bedeutung der zu regelnden Angelegenheit und den Grad der Behinderung bzw. Krankheit des Betroffenen ${ }^{12}$. Ganz generell kann die Bestellung eines Verfahrenspflegers nach \276 Abs. 4 FamFG unterbleiben oder aufgehoben werden, wenn die Interessen des Betroffenen von einem Rechtsanwalt oder einem anderen geeigneten Verfahrensbevollmächtigten vertreten werden.

b) Bestellung in Zeiten von COVID-19

Auf die Bestellung des Verfahrenspflegers kann einfacher verzichtet werden, da das Gesetz keine obligatorische Bestellung des Verfahrenspflegers vorsieht, sondern es immer auf den jeweiligen Einzelfall ankommt. Da mittlerweile anerkannt ist, dass dem Verfahrenspfleger eine besondere Rolle im Betreuungsverfahren zukommen kann, sollte jedoch nicht vorschnell von einer Bestellung abgesehen werden. In den vergangenen Zeiten stand das objektive Interesse des Betroffenen im Vordergrund der Aufgabenwahrnehmung des Verfahrenspflegers. Heute wird dem Verfahrenspfleger aufgrund des Art. 12 Abs. 4 UN-BRK zudem verstärkt die Aufgabe übertragen, die subjektiven Vorstellungen und Wünsche des Betroffenen in das Verfahren einzubringen. Auf diese Weise werde dem Betroffenen die Teilnahme am Verfahren wieder ermöglicht, indem der Verfahrenspfleger seine eigenen Beteiligungsrechte für den Betroffenen ausübt. Der Verfahrenspfleger übernimmt eine Mittlerrolle zwischen dem Betroffenen und den anderen am Verfahren beteiligten Personen ${ }^{13}$. Zudem soll der Verfahrenspfleger auf die Einhaltung der verfahrensrechtlichen Vorschriften achten. Daher ist die Bestellung des Verfahrenspflegers sogar zurzeit sehr wichtig; insbesondere dann, wenn das Gericht von einer persönlichen Anhörung des Betroffenen absehen möchte. Auch der Verfahrenspfleger selbst kann sich schützen, indem die hygienischen Schutzmaßnahmen ergriffen werden. Zudem hat der Verfahrenspfleger auch die Möglichkeit, sich telefonisch mit dem Betroffenen in Verbindung zu setzen. Das Gesetz schreibt dem Verfahrenspfleger ja gerade nicht vor, auf welche Art und Weise er den Kontakt mit dem Betroffenen gestalten muss. Das Gericht sollte bei der Bestellung des Verfahrenspflegers einerseits darauf achten, dass der Verfahrenspfleger nicht selbst mit dem Coronavirus infiziert ist und andererseits nicht zur Risikogruppe gehört.

\section{Pflichten des Betreuers}

Ist ein Betreuer einmal bestellt, kennt das Gesetz einige Pflichten, die der Betreuer zu erfüllen hat. Dazu gehört auch die Pflicht zur Förderung der Gesundheit des Betreuten, welche in $\$ 1901$ Abs. 4 S. 1 BGB besonders hervorgehoben wird. Danach sind Betreuer verpflichtet, innerhalb ihres Aufgabenkreises dazu beizutragen, Krankheiten des Betreuten zu beseitigen, zu bessern, ihre Verschlimmerung zu verhüten oder die Folgen zu mildern. Zu beachten ist auch, dass sich ein Betreuer mit dem Betreuten zu besprechen hat bevor er wichtige Angelegenheiten erledigt, \1901 Abs. 3 S. 3 BGB. Insoweit bedarf es des persönlichen Kontakts. Die Pflicht zur persönlichen Besprechung folgt aus dem Prinzip der persönlichen Betreuung ${ }^{14}$. Allerdings gibt es keine festen Vorgaben zur Häufigkeit und zur Art und Weise des persönlichen Kontakts.

\section{Allgemeine Pflichten}

a) Persönlicher Kontakt

Die rechtliche Betreuung erfordert einen fortlaufenden persönlichen Kontakt zwischen dem Betreuer und dem Betroffenen, \1901 Abs. 1 BGB. Das Gesetz schreibt zwar im Gegensatz zum Vormundschaftsrecht nicht vor, wie häufig ein persönlicher Kontakt zwischen Betreuer und Betroffenem stattfinden soll, vgl. $₫ 1793$ Abs. 1a BGB. Es gebietet sich aber, ähnliche Kontakte zu pflegen, das heißt, in relativ kurzen Zeitabschnitten sollte der persönliche Kontakt stattfinden.

\section{b) Aufgabenkreis Gesundheitsfürsorge im Besonderen}

In erster Linie betrifft der Aufgabenkreis der Gesundheitsfürsorge beispielsweise den Abschluss von Behandlungsverträgen und die Einwilligung in bestimmte ärztliche Maßnahmen ${ }^{15}$. Allerdings zählen zur Gesundheitsbetreuung darüber hinaus auch die Organisation der Gesundheitsfürsorge und die Sicherstellung, dass der Betreute die für sein gesundheitliches Wohl nötigen Untersuchungen und Heilbehandlungen tatsächlich erhält und verträgt, vgl. \1901 Abs. $4 \mathrm{BGB}^{16}$. Die tatsächliche Fürsorge für den Betroffenen, seine körperliche Pflege, Versorgung mit Lebensmitteln oder auch die Aufsicht über ihn gehören indes grundsätzlich nicht zu den Aufgaben des Betreuers ${ }^{17}$. Sind nötige tatsächliche Hilfen nicht rechtzeitig zu erlangen, so ist aber auch der Betreuer selbst gehalten, im Rahmen des ihm Zumutbaren für den Betreuten tätig zu werden ${ }^{18}$.

\section{c) Aufgaben und Pflichten in Zeiten der COVID-19 Pandemie}

Inwieweit diese Pflichten in Zeiten der COVID-19 Pandemie weiterhin gelten, ist fraglich. Einerseits spricht das Infektionsrisiko gegen einen persönlichen Kontakt. So spricht sich auch der Berufsverband der Betreuer (BdB) für eine Einschränkung des persönlichen Kontakts aus ${ }^{19}$. Andererseits gilt zu bedenken, dass es Betroffene geben kann, die gerade während der Pandemie auf vermehrte Unterstützung angewiesen sind. Dies betrifft vor allem Menschen, die alleine wohnen oder zusammen mit einem ebenfalls hilfebedürftigen Partner. Diese Menschen sind von den bestehenden Kontaktsperren am meisten betroffen, da Besuche von Angehörigen und Freunden gemäß der BundLänder-Vereinbarung zur Bekämpfung des Coronavirus auf das notwendige Minimum zu reduzieren sind. Einige Bundesländer haben dazu weitergehende Regelungen getroffen, die zusätzliche Ausgangsbeschränkungen vorsehen und ein Verlassen der Wohnung nur aus „triftigen Gründen“" erlauben. Dabei sind es gerade betreuungsbedürfti-

9) BGH, FamRZ 2014, 1446; BGH, NJW 2016, 1828

10) BGH, FamRZ 2017, 1865; BGH, FamRZ 2018, 1692.

11) Bienwald, in: Bienwald/Sonnenfeld/Harm, 6. Aufl. 2016, \276 FamFG, Rdnr. 44.

12) Schneider, in: MüKo/BGB, 8. Aufl. 2020, \$1896, Rdnr. 169.

13) Leeb/Weber, NJOZ 2014, 1201, 1207.

14) Schneider, in: MüKo/BGB, 8. Aufl. 2020, \1901, Rdnr. 23.

15) Vgl. ausdrücklich BT-Dr. 18/13176, S. 5; Forderung bei Riedel/ Stolz, BtPrax 2008, 233, 236.

16) Schneider, in: MüKo/BGB, 8. Aufl. 2020, \1896, Rdnr. 89; Bienwald, in: Bienwald/Sonnenfeld/Harm, 6. Aufl. 2016, \$1896 BGB, Rdnr. 157, Stichwort: Ambulante Dienste.

17) OLG Schleswig, FamRZ 1998, 1259; LG Koblenz, FamRZ 1998, 495, 496; LG Koblenz, FamRZ 2004, 220.

18) Schneider, in: MüKo/BGB, 8. Aufl. 2020, §1896, Rdnr. 89.

19) BdB, Informationen zum Corona-Virus: https://bdb-ev.de/304 Informationen_zum_Corona-Virus.php (zuletzt abgerufen am 14. 4. 2020). 
ge Menschen, die in der eigenen Häuslichkeit leben, die durch den regelmäßigen Kontakt mit einem Betreuer profitieren könnten. Dieser kann zum einen die Organisation des alltäglichen Lebens übernehmen, z.B. das Bestellen von Lieferdiensten, und zum anderen kann der Betreuer dadurch auch dazu beitragen, mögliche Infektionen schneller zu erkennen. Dafür ist ja nicht zwangsläufig der persönliche Kontakt erforderlich, ein kurzes Telefonat oder Videoanrufe zwei- oder dreimal pro Woche könnten schon ausreichend sein, um zu hören, ob der Betroffene hustet oder angeschlagen wirkt. Auf diese Weise käme der Betreuer seiner Verpflichtung zur Förderung der Gesundheit des Betroffenen gemäß $\$ 1901$ Abs. 4 S. 1 BGB nach und würde zudem noch zur Prävention einer weitergehenden Ausbreitung von COVID-19 beitragen. Kommen Telefonund Videoanrufe dagegen aufgrund der gesundheitlichen Einschränkungen des Betroffenen nicht in Betracht, z.B. wegen starker Schwerhörigkeit, sollte der Betreuer trotz des bestehenden Infektionsrisikos den persönlichen Kontakt suchen. Es kommt daher auch hier auf den Einzelfall an. Insbesondere sollte hygienische Schutzkleidung daher zwingend auch Betreuern zur Verfügung gestellt werden. Sofern Betreute in einer Wohneinrichtung leben, können Betretungsverbote für Betreuer bestehen. Allerdings können auch dort Ausnahmen unter Beachtung von Hygieneund Schutzmaßnahmen zugelassen werden. Erfordert die Wahrnehmung der rechtlichen Betreuung einen persönlichen Besuch durch den Betreuer, so kann die Einrichtungsleitung eine Ausnahmegenehmigung erteilen ${ }^{20}$.

\section{Besondere Pfichten im Falle einer Infizierung des Betreuten}

Darüber hinaus können sich spezielle Pflichten für den Betreuer ergeben, wenn sich der Verdacht einer Infizierung beim Betreuten ergibt. Zwar bestehen keine originären Meldepflichten auf Grundlage des Infektionsschutzgesetzes (IfSG), jedoch ist es Aufgabe des Betreuers, die nun erforderlichen medizinischen Maßnahmen zu veranlassen oder die dazu nötige Unterstützung zu gewährleisten, sollte der Betreute nicht selbst dazu in der Lage sein. Die Handlungspflicht des Betreuers ergibt sich insofern aus dem Aufgabenkreis der Gesundheitsfürsorge sowie der allgemeinen Pflicht zur Förderung der Gesundheit des Betreuten gemäß \1901 Abs. 4 S. 1 BGB.

\section{a) Auskunfts- und Unterstützungspflichten}

Bei Anzeichen für eine Infektion des Betreuten kann die zuständige Verwaltungsbehörde gemäß \16 IfSG allgemeine Maßnahmen zur Abwendung drohender Gesundheitsgefahren treffen. Betroffene Personen sind in diesem Fall dazu verpflichtet, bei der Umsetzung der Verhütungsmaßnahmen mitzuwirken. \16 Abs. 2 S. 2 und 3 IfSG normieren insofern eine Pflicht zur Zugänglichmachung von Grundstücken, Räumen oder sonstigen Gegenständen sowie zur Erteilung von Auskünften. Besteht für den Betroffenen eine rechtliche Betreuung mit entsprechendem Aufgabenkreis, so ist der Betreuer für die Erfüllung dieser Auskunfts- und Unterstützungspflichten gegenüber der Verwaltungsbehörde verantwortlich, \16 Abs. 5 IfSG.

\section{b) Ärztliche Maßnahmen}

Zudem können ärztliche Behandlungen notwendig werden, wenn ein Infektionsverdacht besteht oder der Betreute positiv auf das Coronavirus getestet wurde. Wehrt sich dieser gegen die erforderlichen ärztlichen Maßnahmen und kann er die Notwendigkeit der medizinischen Behandlung aufgrund einer geistigen oder seelischen Behinderung nicht einsehen, so ist eine ärztliche Zwangsbehandlung zu erwägen.

Diesbezüglich kann das Gesundheitsamt krankheitsverdächtige Personen gemäß $\$ 25$ Abs. 3 S. 2 Nr. 1 IfSG zur
Duldung von Untersuchungen und Entnahmen von Untersuchungsmaterial verpflichten (z.B. Blutentnahmen oder Abstriche von Haut und Schleimhäuten).

Sollen jedoch tiefgreifendere Behandlungen gegen den natürlichen Willen des Betroffenen durchgeführt werden, so ist der Betreuer gehalten, in eine ärztliche Zwangsbehandlung gemäß §1906a BGB einzuwilligen, vgl. \\$ 25 Abs. 3 S. 3, 16 Abs. 5 IfSG. Dazu bedarf er der Genehmigung des Betreuungsgerichts, $\$ 1906$ a Abs. 2 BGB. Dieses hat sich im Rahmen des gerichtlichen Verfahrens vom Vorliegen der materiell-rechtlichen Voraussetzungen für die ärztliche Zwangsbehandlung zu überzeugen. Dazu ist gemäß den $\$ \$ 312$ ff. FamFG grundsätzlich die Anhörung des Betroffenen, die Einholung eines Sachverständigengutachtens und die Bestellung eines Verfahrenspflegers vorgesehen. Insofern stellen sich mit Blick auf eine mögliche Ansteckungsgefahr dieselben Probleme wie im Verfahren zur Betreuerbestellung (vgl. die Ausführungen unter I.). Im Ergebnis darf jedoch auch im betreuungsrechtlichen Genehmigungsverfahren nicht allein aufgrund des Vorhandenseins einer möglichen Infektion mit dem Coronavirus von der ordnungsgemäßen Durchführung des Verfahrens abgesehen werden. Auch hier ist eine sorgfältige Prüfung der Ausnahmevorschriften im Einzelfall geboten.

\section{c) Freiheitsentziehende Maßnahmen}

Darüber hinaus können sich Schwierigkeiten ergeben, wenn die betreute Person positiv auf Corona getestet wurde und nicht einsehen kann, dass sie die eigene Häuslichkeit oder das Zimmer in der Wohneinrichtung nicht mehr verlassen darf. In einem solchen Fall kann die Vornahme einer freiheitsentziehenden Maßnahme zur Verhinderung einer weiteren Verbreitung des Virus in Erwägung gezogen werden, z.B. durch Einsperren im eigenen Zimmer. Hierbei erfolgt die freiheitsentziehende Maßnahme allerdings nicht auf betreuungsrechtlicher Grundlage gemäß $\$ 1906$ Abs. 4 BGB. Zweck der Maßnahme ist gerade nicht die Verhinderung einer Selbstschädigung zum Wohl des Betroffenen, sondern vielmehr der Schutz Dritter vor einer Infizierung, weshalb ein Eingriff nach öffentlich-rechtlichen Regelungen erfolgt. Die einschlägigen Ermächtigungsgrundlagen für eine Ausgangsbeschränkung oder Absonderung finden sich in den $\int S 28$ Abs. 1 S. 1, 30 IfSG.

Für diese gilt nach $\$ 30$ Abs. 2 IfSG das Verfahren in Freiheitsentziehungssachen gemäß os 415 ff. FamFG. Dazu ist ebenfalls grundsätzlich die Bestellung eines Verfahrenspflegers sowie eine persönliche Anhörung des Betroffenen durchzuführen. Allerdings kann das Gericht in diesem Verfahren ausnahmsweise von der persönlichen Anhörung absehen, wenn der Betroffene an einer übertragbaren Krankheit im Sinne des Infektionsschutzgesetzes leidet, $\$ 420$ Abs. 2 Alt. 2 FamFG. Die Ausnahmevorschrift darf jedoch nicht so verstanden werden, dass in Freiheitsentziehungssachen nach dem IfSG generell auf die persönliche Anhörung des Betroffenen verzichtet werden $\mathrm{kann}^{21}$. Bestehen Möglichkeiten, um einen ausreichenden Schutz der Gesundheit des anhörenden Richters zu gewährleisten, ist der Betroffene gleichwohl anzuhören ${ }^{22}$. Eine die Anhörung ausschließende Ansteckungsgefahr ist in jedem Fall durch ein ärztliches Gutachten zu belegen, \420 Abs. 2 FamFG. Die Beweisbedürftigkeit einer konkreten Ansteckungsgefahr entfällt auch nicht allein aufgrund der offenkundigen Tatsache, dass aktuell eine allgemein gegenwärtige Infektionsgefahr mit dem Coronavirus gegeben ist, vgl. \$291 ZPO²3.

20) Vgl. z. B. $\$ 2$ Abs. 2 Coronaschutzverordnung des Landes NRW.

21) Göbel, in: Keidel, 20. Aufl. 2020, \$420 FamFG, Rdnr. 12.

22) BGH, FamRZ 2017, 1854

23) A. A. AG Brandenburg v. 6.4.2020 - 85 XVII 69/20, juris. 


\section{Aufgaben des Betreuungsgerichts}

Dem Betreuungsgericht kommt eine besondere Rolle bei der Kontrolle des Betreuers zu.

Nach SS1908i Abs. 1 S. 1, 1837 Abs. 1, 2 BGB hat das Betreuungsgericht den Betreuer nicht nur zu beraten, sondern auch Aufsicht über die Betreuung zu führen. Der Betreuer ist nach $\int S 1908$ i Abs. 1 S. 1, 1839 BGB verpflichtet, Auskunft über die Führung der Betreuung und die persönlichen Verhältnisse des Betreuten zu erteilen. Gemäß \1840 Abs. 1 S. 2 BGB muss der Bericht eine explizite Stellungnahme zum persönlichen Kontakt zwischen dem Betreuer und den Betroffenen enthalten. Das Betreuungsgericht beaufsichtigt daher auch die Einhaltung des persönlichen Kontakts, \$1837 Abs. 2 S. 2 i. V. mit \$1908i Abs. 1 S. 1 BGB. Klar ist, dass der mangelnde Kontakt zwischen Betreuer und Betroffenen einen Entlassungsgrund darstellt, \1908b Abs. 1 S. 2 Alt. 2 BGB. Schwieriger ist indes, ob sich hieraus auch eine Befugnis ergibt, konkrete Kontakte anzuordnen. Einerseits lehnte dies das LG Nürnberg-Fürth $\mathrm{ab}$, da das Gesetz hierfür keine ausreichende Grundlage biete $^{24}$. Andererseits kann das Betreuungsgericht gegen Pflichtwidrigkeiten des Betreuers mittels geeigneten Geboten und Verboten einschreiten. Eine Pflichtwidrigkeit kann insofern in dem unterlassenen persönlichen Kontakt liegen. In diesem Zusammenhang könnte es dann erlaubt sein, die Einhaltung des regelmäßigen Kontakts - zurzeit auch des telefonischen Kontakts - zu gebieten, $\int \$ 1908 i, 1837$ Abs. 2 S. 1, 3 BGB.

\section{Fazit}

Die Besorgnis, sich mit dem Virus zu infizieren, mag verständlich sein, rechtfertigt aber nicht, auf die Einhaltung sämtlicher verfahrens- und materiell-rechtlicher Vorschriften pauschal und generalisierend zu verzichten. Das Gesetz sieht hierfür hohe Hürden vor. Entscheidend kann daher immer nur der jeweilige Einzelfall sein. Generell gilt, dass der persönliche Kontakt nicht vorschnell abgebrochen werden sollte. Vor allem in Zeiten einer Pandemie können betreute Personen verstärkt auf die Hilfe ihres rechtlichen Betreuers angewiesen sein. Diesen kommt eine wesentliche Rolle sowohl bei der Vorbeugung einer möglichen Ansteckung des Betreuten als auch bei dessen Unterstützung im Falle einer Infektion mit dem Coronavirus zu.

24) LG Nürnberg-Fürth, BtPrax 2015, 50 ff.

\title{
Grundrechte in Zeiten von Corona
}

\author{
Zugleich Anmerkung zu BVerfG, Beschl. v. 7.4.2020 - 1 BvR 755/20

\section{Christian Katzenmeier}

\section{Eilrechtsschutz und Hauptverfahren}

In Zeiten der Corona-Pandemie erfolgen massive Grundrechtseinschränkungen. Deren Verfassungskonformität ist hochstreitig. Bevölkerung wie Fachkreise warten auf einen Richterspruch aus Karlsruhe, der für Rechtsklarheit und Rechtssicherheit sorgt. Die erste inhaltlich begründete Entscheidung des BVerfG erging im Eilverfahren. Nach $\$ 32$ Abs. 1 BVerfGG kann das Gericht eine vorübergehende Anordnung zur Vermeidung schwerwiegender Nachteile erlassen, um irreversible Folgen bis zu einer Entscheidung im Hauptsacheverfahren zu vermeiden. Dabei wendet es eine besondere Prüfungsmethode an: die Folgenabwägung nach der sog. Doppelhypothese. Es werden die Nachteile, die eintreten würden, wenn eine Anordnung des Gerichts nicht erginge, das Hauptverfahren jedoch Erfolg hätte, gegen die Nachteile, die entstünden, wenn die begehrte Anordnung des Gerichts erlassen würde, das Hauptsacheverfahren aber später erfolglos bliebe, abgewogen. Das Ergebnis lautet: „Gegenüber den Gefahren für Leib und Leben wiegen die Einschränkungen der persönlichen Freiheit weniger schwer"1 und so wurde der Antrag auf Erlass einer einstweiligen Anordnung - wie nahezu einhellig erwartet - abgelehnt. Damit ist aber nicht gesagt, dass die weitreichenden Einschränkungen von Grundrechten in der Corona-Krise verfassungsgemäß sind. Die Entscheidung in der Hauptsache kann naturgemäß anders ausfallen als die im Eilrechtsschutz.

Prof. Dr. iur. Christian Katzenmeier,

Universität zu Köln, Institut für Medizinrecht,

Albertus-Magnus-Platz, 50923 Köln, Deutschland

\section{Verfassungsbeschwerde}

1. Formelle Verfassungsmäßigkeit von Kontaktverboten/Ausgangsbeschränkungen

Bei „,eingehenderer Prüfung“ der Verfassungsbeschwerde ${ }^{2}$ wird das BVerfG zunächst die formelle Verfassungsmäßigkeit der getroffenen Maßnahmen zu klären haben, im Besonderen die Frage nach der Ermächtigungsgrundlage.

a) IfSG als Ermächtigungsgrundlage

Um die Corona-Krise aufzufangen, wurde am 25.3.2020 im Bundestag ein ganzes Paket an Gesetzen verabschiedet. Der Bundesrat stimmte in einer Sondersitzung am 27.3. 2020 ohne Aussprache zu, noch am selben Tag wurden die Gesetze im Bundesgesetzblatt verkündet. Zentrales Gesetz zur Bekämpfung der Corona-Epidemie ist das Infektionsschutzgesetz (IfSG), das einige Änderungen erfuhr ${ }^{3}$. Gem. $₫ 5$ Abs. 1 S. 1 IfSG kann nun der Deutsche Bundestag eine ,epidemische Lage von nationaler Tragweite" ausrufen. Die aus der epidemischen Lage folgenden Kompetenzen liegen nach $₫ 5$ Abs. 2 IfSG beim Bundesministerium für Gesundheit, das zu weitgehenden Anordnungen und zum Erlass von Rechtsverordnungen ermächtigt wird - ohne Zustimmung des Bundesrates.

1) BVerfG, Beschl. v. 7.4.2020 - 1 BvR 755/20, Rdnr. 11

2) BVerfG, Beschl. v. 7. 4.2020 - 1 BvR 755/20, Rdnr. 7.

3) Durch das ,Gesetz zum Schutz der Bevölkerung bei einer epidemischen Lage von nationaler Tragweite" v. 27.3.2020, BGB1. I S. 587 ff.; dazu Rixen NJW 2020, $1097 \mathrm{ff}$ 